\title{
Increased absorption in Autonomous Sensory Meridian Response
}

\author{
Agnieszka Barbara Janik McErlean ${ }^{\text {Corresp., } 1}$, Eleanor Jane Osborne-Ford ${ }^{1}$ \\ ${ }^{1}$ School of Science, Bath Spa University, Bath, United Kingdom \\ Corresponding Author: Agnieszka Barbara Janik McErlean \\ Email address: a.janikmcerlean@bathspa.ac.uk
}

Background. Autonomous sensory meridian response (ASMR) is a cross-sensory phenomenon characterised by a static-like sensation which typically originates on the scalp and spreads throughout the body leading to a state of deep relaxation. It can be triggered by visual and auditory stimuli in real life, incidentally by various media and via intentionally created ASMR videos. Previously ASMR has been linked to a specific personality profile and this study aimed to further elucidate individual differences associated with this phenomenon. Methods. To this effect ASMR-Experiencers and age and gender matched controls were compared on measures of flow, absorption and mindfulness. Results. This revealed that ASMR was associated with elevated absorption but no group differences were found with respect to the other constructs, suggesting that the ability to get deeply immersed with the current experience accompanied by loss of reflective awareness may be an important factor contributing to the experience of ASMR. 
1 Increased absorption in Autonomous Sensory Meridian Response.

2

3 Agnieszka Barbara Janik McErlean ${ }^{1}$ and Eleanor Jane Osborne-Ford ${ }^{1}$

4 1. School of Science, Bath Spa University, Bath, UK

5

6 Corresponding Author:

7 Dr Agnieszka Janik McErlean

8 School of Science

9 Bath Spa University

10 Bath

11 UK

12 E-mail: a.janikmcerlean@bathspa.ac.uk

13 Tel: +44(0)1225 876637

14

15

16

17

18

19

20

21

22

23

24 Abstract 
25 Background. Autonomous sensory meridian response (ASMR) is a cross-sensory phenomenon

26 characterised by a static-like sensation which typically originates on the scalp and spreads

27 throughout the body leading to a state of deep relaxation. It can be triggered by visual and

28 auditory stimuli in real life, incidentally by various media and via intentionally created ASMR

29 videos. Previously ASMR has been linked to a specific personality profile and this study aimed

30 to further elucidate individual differences associated with this phenomenon. Methods. To this

31 effect ASMR-Experiencers and age and gender matched controls were compared on measures of

32 flow, absorption and mindfulness. Results. This revealed that ASMR was associated with

33 elevated absorption but no group differences were found with respect to the other constructs,

34 suggesting that the ability to get deeply immersed with the current experience accompanied by

35 loss of reflective awareness may be an important factor contributing to the experience of ASMR.

\section{Introduction}

44 Autonomous sensory meridian response (ASMR) is a multisensory phenomenon where

45 auditory and visual stimuli such as whisper or personal attention trigger a pleasant, static-like 
46 tingling sensation which typically originates from the head and disperses throughout the body

47 resulting in a relaxed state (Barratt \& Davis, 2015). Although ASMR can be experienced in

48 daily life, in recent years many ASMR-inducing YouTube channels have been created attracting

49 large audiences who watch such videos to experience the sensation, relax, and fall asleep and in

50 some cases to alleviate anxiety and stress (Barratt \& Davis, 2015; Janik McErlean \& Banissy,

51 2017; Poerio, Blakey, Hostler, \& Veltri, 2018). Although the prevalence of ASMR is not known,

52 the popularity of ASMR media suggests that the phenomenon might be widespread.

53 One line of evidence suggests that there are personality differences between people who

54 experience ASMR and those who do not. Specifically, trait ASMR has been linked to i)

55 increased openness to experience (Janik McErlean \& Banissy, 2017; Fredborg, Clark, \& Smith,

56 2017) which taps into one's interest in novel experiences and propensity to fantasise (John,

57 Naumann, \& Soto, 2008), ii) heightened fantasising (Janik McErlean \& Banissy, 2017), which

58 reflects an ability to immerse oneself in a fictional reality (Davis, 1983), and iii) elevated

59 mindfulness (Fredborg, Clark and Smith, 2018), which entails concentrating on the present

60 moment (Brown \& Ryan, 2003). These findings are interesting taking into account that for many

61 individuals ASMR is triggered when focusing on the external triggers which resembles

62 mindfulness practice (Fredborg et al., 2018) and that ASMR videos, which often entail role

63 plays, require imaginatively transposing oneself into the virtual reality (Janik McErlean \&

64 Banissy, 2017).

ASMR has also been previously likened to a state of flow (Barratt \& Davis, 2015), which can be measured both as a global construct and in terms of its underlying components including warped passing of time (Ross \& Keiser, 2014), complete absorption (Jackson, Thomas, Marsh, \& Smethurst, 2001), and intense concentration when fully engaged in optimally challenging and intrinsically rewarding tasks (Csikszentmihalyi \& Csikszentmihalyi, 1992). Flow has also been 
70 conceptualised as a trait, whereby individuals with the so called autotelic personality (who are

71 intrinsically motivated) are more susceptible to experiencing flow (Csikszentmihalyi, 2000). To

72 date several measures have been developed which allow for measuring flow both as a state and a

73 trait (e.g. Jackson \& Eklund, 2002, 2004). Barratt and Davis (2015) suggested that ASMR is a

74 'flow-like' phenomenon achieved by watching others in a similar state. Interestingly, some of the

75 most popular ASMR triggers, such as watching someone making expert hand movements, are

76 typical examples of being in a state of flow (Janik McErlean \& Banissy, 2017). Moreover,

77 individuals with greater susceptibility to flow have been found to report a greater number of

78 ASMR triggers highlighting a link between the two phenomena (Barratt \& Davis, 2015).

79 Furthermore, a positive association between flow and a newly developed ASMR measure has

80 been recently reported (Roberts, Beath, \& Boag, 2019). However, whether ASMR is associated

81 with increased levels of flow both in terms of intensity and prevalence compared to the general

82 population is currently not known. In addition, both Barratt and Davis (2015) and Roberts et al.,

83 (2019) used a modified version of the Flow State Scale (Jackson \& Marsh, 1996) which only taps

84 into the passive aspects of flow. While this measure is more likely to be relevant in the ASMR

85 context which does not entail active engagement in activities, it does not capture the other core

86 components of the flow experience such as the balance between challenge and skill. As such no

87 study to date examined whether ASMR is associated with increased levels of flow using the

88 global measure of this construct.

89 The reported link between ASMR and flow is also interesting in the context of increased

90 mindfulness among ASMR-experiencers (Fredborg et al., 2018). Mindfulness, similarly to flow,

91 can be conceptualised as a trait and as a state. In Fredborg et al., (2018) study ASMR-

92 experiencers were found to score higher than controls in terms of trait mindfulness based on their

93 scores on the Mindful Attention Awareness Scale which taps into one’s general disposition to pay

94 attention to and be aware of the present moment (Brown \& Ryan, 2003). They also scored higher 
95 on the Curiosity subscale of the state mindfulness measure called the Toronto Mindfulness Scale

96 which suggests increased interest in one’s own inner experiences among the ASMR group (Lau

97 et al., 2006). Although mindfulness and flow are similar in terms of concentrating on the present

98 experience, they differ dramatically in terms of the role of self-awareness which is enhanced in

99 mindfulness and diminished in flow where loss of self-consciousness is the central feature

100 (Bishop, 2002; Bishop et al., 2004; Nakamura \& Csikszentmihalyi, 2009). Moreover, although

101 existing research provides support for the positive association between the global measure of

102 flow comprising all underlying dimensions and mindfulness (Thienot et al., 2014), recent findings

103 suggest that this is driven only by the control facet of flow which reflects a sense of agency and

104 mastery over a task, whereas absorption which is another core underlying dimension of flow is in

105 fact negatively related to mindfulness (Sheldon, Prentice, \& Halusic, 2015).

106 Absorption as a key facet of flow is a trance-like state of consciousness characterised by an

107 ability to fully focus one's attention on a particular object or situation and to become

108 perceptually engrossed with the current experience (Tellegen, 1981, 1982; Tellegen \& Atkinson,

109 1974). It is also a stable personality trait typically measured with the Tellegen Absorption Scale

110 (Tellegen \& Atkinson, 1974). Absorption has also been linked to hypnotisability, imagery, day-

111 dreaming, and openness to experience (Weilbel et al., 2010; Glisky, Tataryn, Tobias, Kihlstrom,

112 \& McConkey, 1991). Recently, an association between absorption propensity and ASMR has

113 also been reported (Roberts et al., 2019). Taking into account existing findings and the

114 phenomenological similarities between absorption and the immersive nature of ASMR, one may

115 expect increased levels of absorption among ASMR-experiencers compared to controls.

116 However, considering previous reports of increased mindfulness in ASMR (Fredborg et al.,

117 2018) and the conceptual incompatibility between mindfulness and absorption (Sheldon et al.,

118 2015) it is essential to examine these constructs together in the context of ASMR. 
119 The purpose of this study was to investigate whether there are differences between ASMR-

120 experiencers and controls in terms of flow, absorption and mindfulness. This study also aimed to

121 elucidate the relationship between these constructs and ASMR characteristics such as intensity,

122 pleasantness, and ASMR videos viewing habits.

\section{Materials \& Methods}

\section{Participants}

125 Participants were recruited via websites dedicated to ASMR and among Psychology students,

126 who were offered credits for their participation. Participants were asked to indicate whether they

127 would classify themselves as controls or ASMR-experiencers based on the description of the

128 phenomenon (as per Janik McErlean \& Banissy, 2017). To further verify that participants in the

129 ASMR group were genuine ASMR-experiencers they were asked to provide a series of answers

130 related to their ASMR experience (see Questionnaire). E.g. they were asked to indicate the

131 intensity of their ASMR when engaging with various stimuli. If a participant indicated they did

132 not experience ASMR in response to any of the popular triggers they were excluded from the

133 analysis. There were only two such individuals, who also happened to be outliers in terms of

134 their age. They were excluded from the analysis. A priori power analysis revealed that suggested

135 sample size to conduct MANOVA was 232 participants in order to obtain statistical power at

1360.90 level $($ Effect size $=0.06, \alpha=0.05)$. Through opportunity sampling, a total of 316

137 participants completed the survey (59\% = ASMR-experiencers). In order to reduce the

138 imbalance between the group sizes and to match the two groups in terms of age and gender 124

139 ASMR-experiencers (92 female, 30 male, 2 other, age $\mathrm{M}=21.79$, SD = 3.36) and 124 controls

140 (92 female, 30 male, 2 other, age $\mathrm{M}=21.40, \mathrm{SD}=3.13$ ) were selected from the total sample

141 prior to data analysis resulting in the final sample of 248 individuals which was used to compare

142 the groups on absorption, flow and mindfulness. These participants were first matched on 
143 gender and then within gender on age +/- 2 years. Majority was matched within $+/-1$ year and

144 four participants who identified as non-binary in terms of gender were matched within +/- 4 145 years.

146 In addition, data contributed by those ASMR-experiencers who stated they watch ASMR

147 videos $(\mathrm{N}=149,121$ female, 26 male, 2 other, age $\mathrm{M}=26.22, \mathrm{SD}=8.12)$ was used in the

148 correlational analysis which aimed to examine the relationship between absorption, flow and

149 mindfulness and ASMR characteristics including pleasantness, intensity, number of videos

150 watched in a single session and frequency of watching ASMR videos.

151

152 Measures

153 The study employed an anonymous survey which was administered via Bristol Online Survey

154 and was approved by the Ethics Reviewers at Bath Spa University. All participants were asked

155 to provide an electronic consent prior to taking this survey.

156 Tellegen Absorption Scale (TAS) employed in this study is a widely used measure of

157 absorption (Tellegen \& Atkinson, 1974). It consists of 34 questions and the participants are

158 asked to indicate the degree to which they agree with each statement such as 'While watching a

159 movie, TV show or a play, I may become so involved that I may forget about myself and my

160 surroundings and experience the story as if it were real and as if I were taking part in it' on a

161 scale from 1 (disagree strongly) to 5 (agree strongly). A total score is calculated by summing the

162 scores for each question. Internal consistency of TAS in this study was $\alpha=.93$.

163 Mindful Attention and Awareness Scale (MAAS) is an established measure of mindfulness

164 (Brown \& Ryan, 2003). It consists of 15 items such as 'I could be experiencing some emotion

165 and not be conscious of it until sometime later'. Participants are asked to indicate how

166 frequently they have such experiences on a scale from 1 (almost always) to 6 (almost never). 
167 The final MAAS score is calculated by averaging all individual answers. The internal

168 consistency of MAAS in this study was $\alpha=.79$.

169 Flow Questionnaire: Firstly, participants were presented with the description of flow (as per

170 Csikszentmihalyi \& Csikszentmihalyi, 1988; Csikszentmihalyi, 1990) based on which they

171 indicated whether they have ever had similar experiences which allows for classifying

172 individuals into those capable of experiencing flow and those who are not. In order to avoid

173 false positive answers those who reported to experience flow were also asked to state what

174 activities they were engaged in when having such experiences. Subsequently, they were asked to

175 complete the Flow Experiences Scale (FES; Schwartz \& Waterman, 2006) which consists of

176 eight statements corresponding to those originally outlined by Csikszentmihalyi and

177 Csikszentmihalyi (1988) and Csikszentmihalyi (1990) which inquire about different aspects of

178 the experience of flow including e.g. 'I lose track of time' to which participants provide a rating

179 on a scale from 1- not at all characteristic of me to 7- very characteristic of me. A total score

180 reflecting the extent to which flow is experienced is calculated by summing the scores for each

181 statement. Question 2 is reverse scored. The internal consistency for FES in this study was $\alpha$ $182=.74$.

183 ASMR Questionnaire: In addition, ASMR-experiencers completed the self-designed ASMR 184 questionnaire which was based on items previously used in ASMR literature (Barratt \& Davis, 185 2015; Janik McErlean \& Banissy, 2017; Fredborg et al., 2018). Specifically, participants were 186 asked: 1) whether they watch ASMR videos (Yes/No), 2) why they watch ASMR videos (open 187 ended), 3) how often they watch ASMR videos: never, less than once a month, 2-3 times a 188 month, 2-3 times a week, daily, 4) whether they require specific conditions to achieve ASMR 189 (open ended), 5) to indicate the intensity of common triggers such as crisp sounds, personal 190 attention etc. using a scale from 0 (no tingles) to 6 (the most intense tingles) - ASMR intensity 191 score was calculated by averaging the scores across all listed triggers as per Fredborg et al. 
192 (2018), 6) whether the intensity of these triggers varies from session to session (Yes/No); 7) to

193 indicate how pleasurable ASMR is from 1- Quite Uncomfortable to 5 - Quite pleasurable. Open

194 ended questions were coded prior to the analysis and the total intensity score was computed by

195 averaging the scores for all listed triggers.

197 Results

198 Data Inspection and Assumptions Testing

199 The data was normally distributed and no outliers were identified as all values fell within +/2002.5 SD from the mean. However, as only 106 out of 248 (43\%) participants reported to 201 experience flow it was not possible to conduct MANOVA due to the insufficient sample size.

202 Instead three separate independent samples t-tests were conducted to compare the groups on the 203 three personality traits.

204

Comparison of ASMR-Experiencers and Controls on Personality Traits

206 Three independent-samples t-tests revealed: i) a non-significant group difference t(104) = -

207 0.990, $\mathrm{p}=.324, \mathrm{~d}=$ 0.19, 95\% CI [-4.807; 1.606] between ASMR-experiencers $(\mathrm{M}=36.49$,

$208 \mathrm{SD}=8.16)$ and controls $(\mathrm{M}=38.09, \mathrm{SD}=8.19)$ in terms of flow, ii) a non-significant group

209 difference on mindfulness $\mathrm{t}(246)=-0.715, \mathrm{p}=.475, \mathrm{~d}=0.09,95 \%$ CI [-0.273; 0.127] between

210 ASMR-experiencers ( $\mathrm{M}=3.20, \mathrm{SD}=0.80)$ and controls $(\mathrm{M}=3.28, \mathrm{SD}=0.79)$, iii) a statistically

211 significant group difference on absorption $\mathrm{t}(246)=4.995, \mathrm{p}<.001, \mathrm{~d}=0.63,95 \% \mathrm{CI}[8.979$;

212 20.252] with ASMR-experiencers (M=108.74, SD=23.80) scoring higher than controls

213 ( $M=94.22, \mathrm{SD}=21.95)$ (see Table 1 for Descriptive Statistics). Furthermore, chi-square analysis

214 revealed that the proportion of individuals who reported to experience flow was significantly

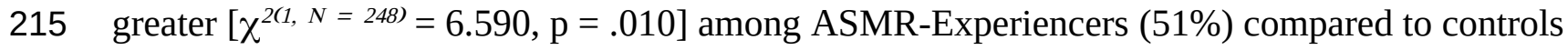
216 (34\%). 
217 An additional analysis was conducted using the whole data set rather than just the subsample

218 of ASMR-experiencers and control participants who were matched for age and gender. This

219 analysis revealed qualitatively similar results to the ones reported above (see Supplemental

220 Results).

221

222

Correlations between ASMR features and personality traits

223 Pearson's correlations employed to examine the relationship between absorption, flow and

224 mindfulness and ASMR characteristics revealed a positive relationship between: i) absorption

225 and the intensity of ASMR $(r(149)=.180, \mathrm{p}=.028)$, ii) intensity and frequency of watching

226 ASMR videos $(\mathrm{r}(149)=.354, \mathrm{p}<.001)$, iii) intensity and pleasantness $(\mathrm{r}(149)=.389, \mathrm{p}<.001)$

227 and iv) pleasantness and frequency of watching ASMR videos $(r(149)=.233, p=.004$. No other

228 correlations were statistically significant (Table 2; Figure 1).

\section{Summary of responses to ASMR Questionnaire}

231 The main motivation behind watching ASMR videos was to relax (71\%), followed by to fall 232 asleep (60.1\%), to experience ASMR (54.1\%), to improve mood, especially in relation to

233 anxiety and depression (12.8\%), and to help concentrate on work or a task (6.8\%). On average, 234 participants watched 3.3 videos per session. 42.3\% reported to watch ASMR videos daily, 235 followed by to 2-3 times a week (30.9\%), 2- 3 times a month (19.5\%), and less than once a 236 month (7.4\%). 49\% of participants stated that they needed specific conditions to experience 237 ASMR. This included: a quiet room (71.23\%), dim lighting (30.14\%), to be alone or have no 238 distractions (26.03\%), to be wearing headphones (20.55\%), to be in bed or lying down (13.7\%), 239 to have the room at a specific temperature (12.33\%) to be in a real life setting (8.22\%), comfort 240 (8.22\%), which is largely consistent with the findings of Barratt, Spence, \& Davis (2017). In 241 terms of the intensity on average whispering $(M=4.03)$ was rated to produce the most intense 
242 ASMR, followed by personal attention $(M=3.65)$, crisp sounds $(M=3.57)$, paying attention to

243 detail, concentrating on something, slowly performing mundane actions or explaining something

$244(M=3.43)$, role-play $(M=3.15)$, hair brushing $(M=3.04)$ and lastly people eating $(M=1.34)$.

$24594 \%$ reported that the intensity of ASMR varies from session to session. The majority of

246 participants (60.4\%) gave ASMR the highest rating in terms of how pleasurable it is, followed

247 by $35.6 \%$ rating ASMR as mildly pleasant, 2.7\% as neutral, one participant reported ASMR to

248 be quite uncomfortable $(0.7 \%)$ and another one as mildly uncomfortable $(0.7 \%)$.

250 Discussion

251 The purpose of this study was to further elucidate whether ASMR is associated with wider

252 differences in terms of personality traits. Current results demonstrate that ASMR-experiencers

253 show elevated absorption compared to age and gender matched controls, but no group

254 differences were found in terms of mindfulness or flow. This suggests that ASMR-experiencers

255 display increased readiness for experiential involvement and heightened ability to become fully

256 engaged with the current experience(Tellegen, 1981; Jamieson, 2005). This is interesting taking

257 into account that being immersed in the virtual reality appears to be a key component of

258 technologically-mediated ASMR and that increased absorption has been previously linked to a

259 more immersive virtual reality experience (Baños et al., 1999). Moreover, increased absorption

260 has been associated with elevated openness to experience (Weilbel, Wissmath, \& Mast, 2010;

261 Glisky et al., 1991) which is also heightened among ASMR-experiencers (Janik McErlean \&

262 Banissy, 2017; Fredborg et al., 2017). ASMR has also been linked to another trait relevant to

263 technologically-mediated ASMR i.e. increased fantasizing (Janik McErlean \& Banissy, 2017)

264 which reflects heightened propensity to become imaginatively involved in a fictional reality. As

265 such, the combination of increased absorption, openness to experience and fantasizing may 
266 contribute to the individual likelihood of experiencing ASMR. Moreover, when considering

267 those individuals who consume ASMR media to alleviate stress and anxiety it is possible that

268 for them becoming fully absorbed in ASMR videos may act as a form of distraction from

269 psychological distress which is similar to the well documented effect of virtual reality gaming

270 and other immersive methods as effective pain reduction strategies (Dahlquist et al., 2007;

271 Jameson, Trevena, \& Swain, 2011).

272 The current study also found that ASMR-experiencers did not differ from controls in terms of

273 mindfulness, which is consistent with our results of increased absorption among ASMR-

274 experiencers and the reports of conceptual incompatibility between the two constructs (Brown \&

275 Ryan, 2003; Sheldon et al., 2015). Moreover, the correlational analysis employed in this study

276 revealed no association between mindfulness and absorption further suggesting little overlap

277 between these psychological variables. It therefore appears that it is the total immersion in the

278 experience accompanied by a loss of self-awareness, which are core features of absorption,

279 rather than sustained consciousness of the current moment characteristic of mindfulness that are

280 features relevant to the ASMR experience. However, it is of note that these results are

281 inconsistent with previous findings of increased mindfulness in ASMR (Fredborg et al., 2018).

282 As both studies employed the same scale and participant recruitment method it is not clear why

283 different results were obtained. Although the sample size in this study was not as large as in the

284 other one, it was sufficiently large as determined by a priori power analysis. However, it appears

285 that the control participants sampled by Fredborg et al., (2018) scored particularly low on

286 MAAS $(\mathrm{M}=3.02, \mathrm{SD}=1.17)$ compared to the control participants in the present study $(\mathrm{M}=$

2873.28 , SD = 0.79), which might explain the difference in results between the two studies.

288 Moreover, both the current and Fredborg et al., (2018)'s results pertaining to the control sample

289 are much lower than originally reported by the MAAS authors who found average MAAS scores 
290 to be $\mathrm{M}=3.85(\mathrm{SD}=0.68)$ among undergraduate students and $\mathrm{M}=3.97(\mathrm{SD}=0.64)$ in a

291 community sample (Brown \& Ryan, 2003). It is not clear what drives this discrepancy in

292 findings between the three studies. As such, the potential link between ASMR and mindfulness

293 should be further explored in future research.

294 Furthermore, current results show similar levels of flow between ASMR-experiencers and

295 controls. This is consistent with existing literature demonstrating an association between the

296 global flow construct and mindfulness (Thienot et al., 2014) and current findings of no group

297 differences on either of these traits. This is also in line with the original conceptualisation of the

298 construct which emphasises that active engagement in activities is necessary for the experience

299 of flow (Csikszentmihalyi \& Csikszentmihalyi, 1992) and research showing that flow has been

300 most commonly related to activities that are effortful and based on challenge, skill and intrinsic

301 motivation (Mauri, Cipresso, Balgera, Villamira, \& Riva, 2011) whilst the experience of ASMR

302 is typically elicited through passively watching videos or by observing people in real life who

303 engage in ASMR-inducing activities. Therefore, ASMR appears to only reflect the passive

304 aspects of flow, which are more consistent with the construct of absorption (Jackson et al.,

305 2001). This is also in line with Barratt and Davis (2015) and Roberts et al., (2019) studies who

306 measured flow in terms of its passive component which was found to be positively associated

307 with the ASMR experience. Moreover, these results also fit with previous reports of elevated

308 fantasising and imaginative involvement in ASMR (Janik McErlean \& Banissy, 2017) and the

309 fact that fantasy engagement and imagination are key characteristics of absorption but not flow

310 (Nakamura \& Csikszentmihalyi, 2014). However, it is of note that substantially more ASMR-

311 experiencers reported to experience flow (51\%) compared to controls (34\%) suggesting that

312 while the extent to which both groups experience flow is similar, flow appears to be more

313 prevalent among ASMR-experiencers. Interestingly, the majority of ASMR sample reported to 
314 experience flow when engaged in sport, music, art or when concentrating on a work-related task

315 which are typical examples of flow-inducing activities (Csikszentmihalyi \& Rathunde, 1993)

316 and only five individuals reported to experience flow when consuming ASMR media. This

317 suggests that ASMR group appears to experience flow more readily than controls but rarely in

318 an ASMR context. However, it is of note that although previous research is quite inconsistent

319 when it comes to the prevalence of flow, the percentage of individuals identifying with the

320 experience in this study (both ASMR-experiencers and controls) is comparably low. For

321 instance, Moneta (2012) reports that about two-thirds of the general population are capable of

322 experiencing flow while some of the earlier studies suggest the experience to be universal with

323 prevalence rates of 97\% among university students (Massimini, Csikszentmihalyi, \& Delle Fave,

324 1988). Although, Han (1988) reports that only 33\% of elderly Korean immigrants in America

325 identified with the experience suggesting potential effects of age and culture.

326 This study also found that absorption was positively correlated with the intensity of ASMR

327 suggesting that this trait is linked to the extent to which ASMR is experienced. However, no

328 association was found between flow and mindfulness and ASMR features, further demonstrating

329 that these traits are not related to ASMR. In addition to this, our results suggest that those who

330 experience ASMR more intensely find it more pleasurable and also engage with ASMR media

331 more frequently. However, it is important to note that due to the correlational nature of this

332 research it is not possible to conclude that the trait of absorption predisposes people to

333 experience ASMR or that the ability to experience ASMR increases the propensity for

334 absorption. It is also possible that both absorption and ASMR are underlined by a third variable

335 such as openness to experience.

336 Furthermore, although one may suspect that the self-selection bias may have contributed to

337 the current results of increased absorption among ASMR-experiencers compared to controls as 
$33875 \%$ of the ASMR sample whose data was used for comparison purposes and overall $80 \%$ of the 339 entire ASMR sample in this study (not just the ones matched for age and gender) reported to 340 watch ASMR-videos. However, we have found that ASMR-experiencers who watch ASMR

341 videos $(\mathrm{M}=108.31, \mathrm{M}=27.27)$ and ASMR-experiencers who do not watch such videos $(\mathrm{M}=$

$342108.04, \mathrm{SD}=25.679$ ) reported exactly the same level absorption suggesting that our results

343 pertain also to those ASMR-experiencers who do not engage with ASMR media.

344 In addition, consistently with previous research this study has found that the key reasons for 345 watching ASMR videos were to relax, fall asleep, to experience ASMR, and to help with stress 346 or anxiety (Barratt \& Davis, 2015; Janik McErlean \& Banissy, 2017). In addition, 6.7\% of

347 participants reported to play ASMR videos in the background while studying or working.

348 Moreover, 49\% of participants stated that they needed specific conditions, such as a quite space 349 with few distractions, in order to experience ASMR which is consistent with the previous 350 studies (Barratt \& Davis, 2015).

\section{Conclusions}

352 In summary, our findings contribute to the existing literature documenting individual 353 differences associated with ASMR by showing that absorption proneness may be an important 354 factor contributing to the experience of ASMR. This study also shows that ASMR is not linked 355 to the constructs of flow and mindfulness. 
358 Baños, R., Botella, C., García-Palacios, A., Villa, H., Perpiñá, C., \& Gallardo, M. (1999).

359 Psychological variables and reality judgment in virtual environments: The roles of absorption 360 and dissociation. CyberPsychology \& Behavior, 2(2), 143-148.

361 Barratt, E. L., \& Davis, N. J. (2015). Autonomous Sensory Meridian Response (ASMR): a flow$362 \quad$ like mental state. PeerJ, 3, e851.

363 Barratt, E., L., Spence, C., \& Davis, N. J. (2017). Sensory determinants of the autonomous 364 sensory meridian response (ASMR): understanding the triggers. PeerJ, 5:e3846.

365 Bishop, S.R. (2002). What do we really know about mindfulness-based stress reduction?

366 Psychosomatic Medicine, 64(1), 71-83.

367 Bishop, S.R., Lau, M., Shapiro, S., Carlson, L., Anderson, N.D., Carmody, J., Segal, Z.V., 368 Abbey, S., Speca, M., Velting, D., \& Devins, G. (2004). Mindfulness: a proposed operational 369 definition. Clinical Psychology: Science and Practice, 11(3), 230-241.

370 Brown, K. W., \& Ryan, R. M. (2003). The benefits of being present: Mindfulness and its role in 371 psychological well-being. Journal of Personality and Social Psychology, 84, 822 - 848.

372 Csikszentmihalyi, M. (1988). The future of flow. In M. Csikszentmihalyi \& I. S.

373 Csikszentmihalyi (Eds.), Optimal experience: Psychological studies of flow in consciousness

374 (pp. 364-383). Cambridge; New York: Cambridge University Press.

375 Csikszentmihalyi, M. (1990). Flow: The psychology of optimal experience. New York: Harper \& $376 \quad$ Row.

377 Csikszentmihalyi, M. (2000). Beyond boredom and anxiety: Experiencing flow in work and play. 378 San Francisco: Jossey-Bass. First published in 1975.

379 Csikszentmihalyi, M., \& Csikszentmihalyi, I.S. (1992). Optimal Experience: Psychological

380 Studies of Flow in Consciousness. Cambridge: Cambridge University Press.

381 Csikszentmihalyi, M., \& Rathunde, K. (1993). The measurement of flow in everyday life. 382 Nebraska Symposium on Motivation, 40, 57-97. 
383 Dahlquist, L. M., McKenna, K. D., Jones, K. K., Dillinger, L., Weiss, K. E., \& Ackerman, C. S. 384 (2007). Active and passive distraction using a head-mounted display helmet: Effects on cold 385 pressor pain in children. Health Psychology, 26(6), 794-801.

386 Davis, M. H. (1983). Measuring individual differences in empathy: Evidence for a 387 multidimensional approach. Journal of personality and social psychology, 44(1), 113 -126. 388 Fredborg, B., Clark, J., \& Smith, S. D. (2017). An examination of personality traits associated 389 with Autonomous Sensory Meridian Response (ASMR). Frontiers in Psychology, 8, 247. 390 Fredborg, B.K., Clark, J.M., \& Smith, S.D. (2018). Mindfulness and autonomous sensory 391 meridian response (ASMR)’’ PeerJ, 6:e5414.

392 Glisky, M.L., Tataryn, D.J., Tobias, B.A., Kihlstrom, J.F., \& McConkey, K.M. (1991). 393 Absorption, openness to experience, and hypnotizability. Journal of Personality and Social $394 \quad$ Psychology, 60(2), 263-272.

395 Han, S. (1988). The relationship between life satisfaction and flow in elderly Korean 396 immigrants. In M. Csikszentmihalyi \& I. S. Csikszentmihalyi (Eds.), Optimal experience: 397 Psychological studies of flow in consciousness. Cambridge; New York: Cambridge $398 \quad$ University Press.

399 Jackson, S. A., \& Eklund, R. C. (2002). Assessing flow in physical activity: The Flow State 400 Scale-2 and Dispositional Flow Scale-2. Journal of Sport and Exercise Psychology, (24), $401 \quad 133-150$.

402 Jackson, S. A., \& Eklund, R. C. (2004). The Flow Scales manual. Morgantown, WV: Fitness 403 Information Technology, Inc.

404 Jackson, S.A., Marsh, H.W., (1996). Development and validation of scale to measure optimal 405 experience: flow state scale. Journal of Sport and Exercise Psychology 18 (1), 17-35. 
406 Jackson, S.A., Thomas, P.R., Marsh, H.W., \& Smethurst, C.J. (2001). Relationship between

407 flow, self-concept, psychological skills and performance. Journal of Applied Sport

408 Psychology, 13(2), 129-153.

409 Jameson, E., Trevena, J., \& Swain, N. (2011). Electronic gaming as pain distraction. Pain

$410 \quad$ Research and Management, 16(1), 27-32.

411 Jamieson, G.A. (2005). The modified tellegen absorption scale: a clearer window on the

412 structure and meaning of absorption. Australian Journal of Clinical and Experimental

413 Hypnosis, 33(2), 119-139.

414 Janik McErlean, A.B. and Banissy, M.J. (2017) 'Assessing individual variation in personality

415 and empathy traits in self-reported autonomous sensory meridian response.' Multisensory

$416 \quad$ Research, 30(6), 601-613.

417 John, O. P., Naumann, L. P., \& Soto, C. J. (2008). Paradigm shift to the integrative big-five trait

418 taxonomy: history, measurement, and conceptual issues. In: O. P. John, R. W. Robins, \& L.

419 A. Pervin (Eds.), Handbook of personality: theory and research (pp. 114-58). New York:

$420 \quad$ Guilford Press.

421 Lau, M. A., Bishop, S. R., Segal, Z. V., Buis, T., Anderson, N. D., Carlson, L., ... \& Devins, G.

422 (2006). The Toronto mindfulness scale: Development and validation. Journal of clinical

423 psychology, 62, 1445-1467. doi: 10.1002/jclp.20326

424 Massimini, F., Csikszentmihalyi, M., \& Delle Fave, A. (1988). Flow and biocultural evolution.

425 In M. Csikszentmihalyi \& I. S. Csikszentmihalyi (Eds.), Optimal experience: Psychological

426 studies of flow in consciousness (pp. 288-306).

427 Mauri, M., Cipresso, P., Balgera, A., Villamira, M., \& Riva, G. (2011). Why is Facebook so

428 successful? Psychophysiological measures describe a core flow state while using Facebook.

429 Cyberpsychology Behaviour and Social Networking, 14(12), 723-731. 
430 Moneta, G. B. (2012). On the measurement and conceptualization of flow. In S. Engeser (Ed.), 431 Advances in flow research (pp. 23-50). New York: Springer.

432 Nakamura, J., \& Csikszentmihalyi, M. (2009). Flow theory and research. In S. Lopez \& C. R. 433 Snyder (Eds.), Oxford handbook of positive psychology (2nd ed., pp. 89-105). New York, 434 NY: Oxford University Press.

435 Nakamura, J., \& Csikszentmihalyi, M. (2014). The concept of flow. In Flow and the 436 foundations of positive psychology (pp. 239-263). Springer, Dordrecht.

437 Poerio, G. L., Blakey, E., Hostler, T. J., \& Veltri, T. (2018). More than a feeling: Autonomous 438 sensory meridian response (ASMR) is characterized by reliable changes in affect and 439 physiology. PloS one, 13(6), 1-18.

440 Roberts, N., Beath, A., \& Boag, S. (2019). Autonomous sensory meridian response: scale 441 development and personality correlates. Psychology of Consciousness: Theory Research, and $442 \quad$ Practice, 6(1), 22-39. https://doi.org/10.1037/cns0000168

443 Ross, S.R., \& Keiser, H.N. (2014). Autotelic personality through a five-factor lens: individual 444 differences in flow propensity. Personality and Individual Differences, 59, 3-8.

445 Schwartz, S.J., \& Waterman, A.S. (2006). Changing interests: a longitudinal study of intrinsic 446 motivation for personality salient activities. Journal of Research in Personality, 40(6), 1119$447 \quad 1136$.

448 Sheldon, K. M., Prentice, M., \& Halusic, M. (2015). The experiential incompatibility of 449 mindfulness and flow absorption. Social Psychological and Personality Science, 6(3), 2762830.

451 Tellegen A. (1982). Brief manual for the Differential Personality Questionnaire. Unpublished 452 manuscript, University of Minnesota. Tellegen, A. (1987, October). Discussion: Hypnosis 453 and absorption. Paper presented at the 38th annual meeting of the Society for Clinical and 454 Experimental Hypnosis, Los Angeles. 
455 Tellegen, A., \& Atkinson, G. (1974). Openness to absorbing and self-altering experiences

456 ("absorption"), a trait related to hypnotic susceptibility. Journal of Abnormal Psychology, 83, $457 \quad 268-277$.

458 Tellegen, A. (1981). Practicing the two disciplines for relaxation and enlightenment: Comment 459 on "Role of the feedback signal in electromyographic biofeedback: The relevance of 460 attention” by Quails and Sheehan. Journal of Experimental Psychology: General, 110, 217461226.

462 Thienot, E., Jackson, B., Dimmock, J., Grove, J. R., Bernier, M., \& Fournier, J. F. (2014). 463 Development and preliminary validation of the mindfulness inventory for sport. Psychology 464 of Sport and Exercise, 15, 72-80.

465 Weilbel, D., Wissmath, B., \& Mast, F.W. (2010). Immersion in mediated environments: the role 466 of personality traits. Cyberpsychology, Behaviour and Social Networking, 13(3), 251-256. 


\section{Table 1 (on next page)}

Means and Standard Deviations for ASMR-Experiencers and Controls on FES, MAAS and TAS

Note: $\mathrm{FES}=$ Flow Experiences Scale, MAAS $=$ Mindful Attention and Awareness Scale, TAS $=$ Tellegen Absorption Scale. $\mathrm{N}$ (MAAS, TAS): $A S M R=124$, Control $=124 ; \mathrm{N}(\mathrm{FES})$ : $\mathrm{ASMR}=63$, Control $=43$. 
1 Table 1

2 Means and Standard Deviations for ASMR-Experiencers and Controls on FES, MAAS and TAS

3

\begin{tabular}{lllll}
\hline Variable & ASMR & \multicolumn{3}{c}{ Control } \\
\cline { 2 - 5 } & Mean & SD & Mean & SD \\
\hline FES & 36.49 & 8.16 & 38.09 & 8.19 \\
MAAS & 3.20 & 0.80 & 3.28 & 0.79 \\
TAS & 108.74 & 23.80 & 94.22 & 21.95 \\
\hline
\end{tabular}

4 Note: $\mathrm{FES}=$ Flow Experiences Scale, MAAS = Mindful Attention and Awareness Scale, TAS =

5 Tellegen Absorption Scale. N (MAAS, TAS): ASMR=124, Control = 124; N (FES): ASMR = $6 \quad 63$, Control $=43$.

7

8

9

10

11 


\section{Table 2 (on next page)}

Correlations between ASMR intensity, number of videos watched in a single session, frequency of watching ASMR videos, pleasantness of ASMR and the scores on TAS, MAAS and FES.

Note: $\mathrm{N}$ (Intensity, Frequency, Pleasantness, TAS ad MAAS) $=149 . \mathrm{N}($ Number $)=143 . \mathrm{N}$ $(\mathrm{FES})=72 . \mathrm{TAS}=$ Tellegen Absorption Scale, MAAS $=$ Mindful Attention and Awareness

Scale, FES $=$ Flow Experiences Scale. ${ }^{*} p<.05,{ }^{* * p}<.01,{ }^{* * *} p<.001$. 
1 Table 2

2 Correlations between ASMR intensity, number of videos watched in a single session, frequency

3 of watching ASMR videos, pleasantness of ASMR and the scores on TAS, MAAS and FES.

\begin{tabular}{llllllll}
\hline Variable & Intensity & Number & Frequency & Pleasantness & TAS & MAAS & FES \\
\hline Intensity & - & -.005 & $.354^{* * *}$ & $.389^{* * *}$ & $.180^{*}$ & .033 & .207 \\
Number & -.005 & - & .111 & -.030 & -.061 & -.040 & -.232 \\
Frequency & $.354^{* * *}$ & .111 & - & $.233^{* *}$ & -.009 & -.072 & .017 \\
Pleasantness & $.389^{* * *}$ & -.030 & $.233^{* *}$ & - & .156 & -.042 & .168 \\
TAS & $.180^{*}$ & -.061 & -.009 & .156 & - & -.027 & .156 \\
MAAS & .033 & -.040 & -.072 & -.042 & -.027 & - & .173 \\
FES & .207 & -.232 & .017 & .168 & .156 & .173 & - \\
\hline
\end{tabular}

4 Note: N (Intensity, Frequency, Pleasantness, TAS ad MAAS) $=149 . \mathrm{N}($ Number $)=143 . \mathrm{N}$

$5 \quad($ FES $)=72$. TAS $=$ Tellegen Absorption Scale, MAAS $=$ Mindful Attention and Awareness

6 Scale, $\mathrm{FES}=$ Flow Experiences Scale. ${ }^{*} p<.05, * * p<.01, * * * p<.001$.

7

8 
Figure 1

Figure 1

A) Relationship between ASMR Intensity and absorption (TAS), mindfulness (MAAS), and flow (FES) based on z scores, B) Relationship between ASMR Intensity and TAS based on original scores, C) Relationship between ASMR Intensity and MAAS based on original scores, D) Relationship between ASMR Intensity and FES based on original scores.

A)

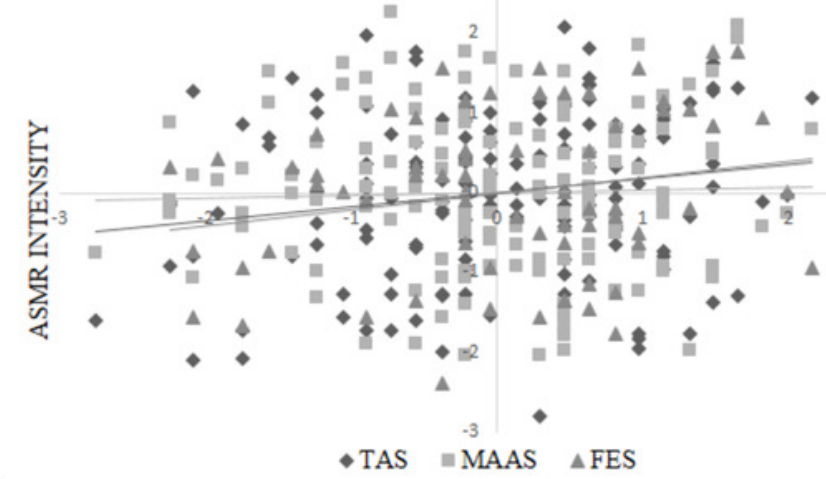

B)

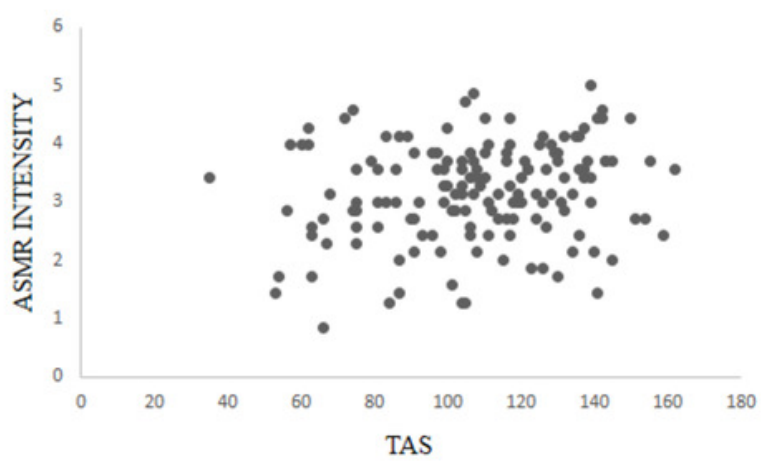

C)

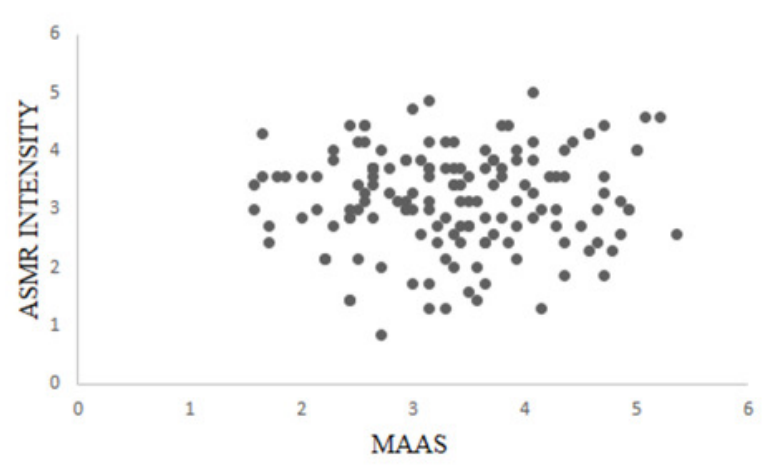

D)

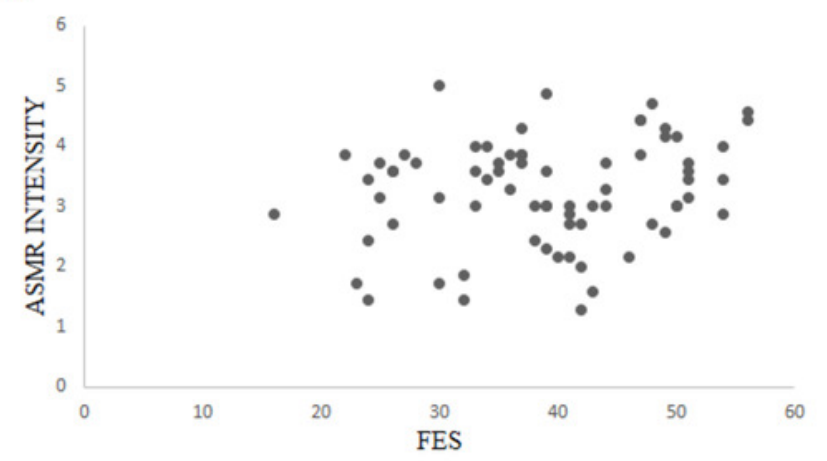

